\title{
Tejiendo masculinidades: tres relatos de vida acerca de la construcción social de la masculinidad en sus narrativas sobre el cuerpo y la indumentaria*
}

\author{
Jesús Donaldo Saucedo Rodríguez ${ }^{1}$
}

\begin{abstract}
Resumen
Los estudios de género han puesto en la mira del análisis social los procesos socioculturales que atraviesan la dimensión simbólica y práctica del género a través del cuerpo. Por ello, se propuso analizar el proceso de construcción de la masculinidad de tres hombres saltillenses con relación a sus narrativas sobre el cuerpo y la indumentaria, a partir de sus relatos de vida y un trabajo visual. El análisis sugiere que la manera en la que estos hombres llevan y adornan su cuerpo cotidianamente narra los procesos de construcción de la masculinidad por los cuales significan su experiencia subjetiva como hombres.

Abstract

Gender studies have focused on social analysis the socio-cultural processes that cross the symbolic and practical dimension of gender throughout the body. Therefore, it was proposed to analyze the process of construction of the masculinity of three saltillenses men in relation to their narratives about the body and the clothing based on their life stories and visual work. The analysis suggests that the way in which these men carry and adorn their body daily narrates the processes of masculinity construction by which they mean their subjective experience as men.
\end{abstract}

\section{Palabras Clave}

Masculinidades, Subjetividades, Visualidad, Cuerpo, Indumentaria.

Keywords

Masculinities, Subjectivities, Visuality, Body, Clothing.

*Primer lugar del concurso de Trabajos Recepcionales en Comunicación CONEICC 2019. Universidad Autónoma de Coahuila.

Directora de tesis: Dra. Adriana Marcela Moreno Acosta.

1 Autor para correspondencia: jesusdonaldo@gmail.com

\section{Objeto de estudio}

¿Qué papel cumple lo sociocultural en el significado de ser y comportarse como un hombre? ¿De qué manera el cuerpo, desde su adorno y movimientos, puede significar un sistema de comunicación capaz de narrar la experiencia subjetiva de cada individuo? ¿Qué relación existe entre la constitución de la cotidianidad y la construcción social del género?

Estas fueron algunas de las interrogantes que ayudaron a sentar una serie de reflexiones en una investigación básica de carácter exploratorio correspondiente al trabajo de tesis
Tejiendo Masculinidades: tres relatos de vida acerca de la construcción social de la masculinidad en sus narrativas sobre el cuerpo y la indumentaria, en donde se indagó acerca del proceso subjetivo bajo el cual tres hombres de la ciudad de Saltillo, Coahuila, construyen socialmente su masculinidad, a partir de sus narrativas sobre el cuerpo y la indumentaria. Para realizar esta investigación se abordaron distintas obras teóricas relacionadas a la comunicación, la sociología, la antropología y los estudios visuales, con el fin de entablar un puente entre los múltiples procesos presentes en la construcción de la masculinidad que realizan estos hombres en su vida cotidiana. 
En este tenor, al indagar en los procesos constituidos en la subjetividad de cada individuo, y de interesarse en observar el papel que toma el cuerpo y la indumentaria en esta construcción social, se decidió encaminar la investigación bajo un enfoque biográfico. Lo anterior, por medio de los relatos de vida (Bertaux, 1989) y la foto-biografía (Cronin, 1998), atribuyendo un papel importante a los relatos hechos por estos hombres, al ser un medio por el cual el lenguaje y la visualidad se presentan como acervos de representaciones simbólicas que son construidos y objetivados en la vida cotidiana en forma de conocimiento (Berger y Luckmann, 2003).

Para Giménez (2011), dentro de los procesos comunicativos "no se transmite sólo un mensaje, sino también una cultura, una identidad y el tipo de relación social que enlaza a los interlocutores" (p. 109). Es decir, la comunicación vista como un proceso, conglomera una serie de atributos que caracterizan a ciertos fenómenos, dando cabida a posibles análisis de los discursos que se encuentran presente en los procedimientos cotidianos.

El trabajo de investigación presentado, se enfocó en el estudio de las masculinidades a través de la construcción social, tratada desde las narrativas del cuerpo y la indumentaria, con la intención de dar una primera exploración al proceso de hacerse hombre; de observar el papel que toma el cuerpo y la vestimenta en la construcción de la masculinidad y el papel que cumple lo sociocultural en dicha construcción.

Se realizó una búsqueda de distintos trabajos de investigación (Zambrini, 2013) que dedicaran sus esfuerzos a estudiar al hombre y la masculinidad a partir de sus visualidades, su cuerpo y narrativas del cuerpo e indumentaria, con el fin de observar el estado en el que se encontraban los estudios de género con relación al construccionismo social, los relatos de vida y la imagen en le investigación social.

Aquí se identificó la relación entre el género y el cuerpo, al ser este último el medio por el cual se ejecutan, se reproducen y se modifican los actos y significados que constituyen al propio género. Esto se pudo comprobar a lo largo de los resultados de las investigaciones referidas, en dónde el cuerpo supone la fuente principal por la que el hombre adquiere, refuerza, demuestra y pierde su masculinidad.

Asimismo, fue posible identificar la relación entre la experiencia corporal y la subjetividad del individuo, pues factores como la clase, la raza, la edad, los consumos, los gustos y los comportamientos en los hombres se encuentran estrechamente vinculados con los ideales que representan la masculinidad en sus vidas. La visualidad supuso otro de los puntos a destacar en esta aproximación: los resultados de diversas investigaciones retomaban el papel que cumple la mirada en la forma en la que el hombre aprende, reproduce y vuelve legitima su masculinidad por medio de la presentación del cuerpo y del performance de género que reproduce en y a través de él (Butler, 1990).

En este tenor, se creyó importante abordar el trabajo de tesis bajo la misma línea de investigación que estudia la masculinidad como un fenómeno construido socialmente, pues las ideas y significados de vivir la masculinidad son distintos en cada hombre, en cada lugar y en cada circunstancia. Lo anterior abre la posibilidad de visualizar no una, sino múltiples masculinidades que se constituyen a la par de la subjetividad de cada individuo, razón por la cual el estudio se apoyó de la siguiente pregunta de investigación: ¿cómo se construye socialmente la masculinidad con relación a las narrativas del cuerpo y la indumentaria de tres hombres saltillenses?

Mientras que el objetivo principal radicó en analizar el proceso de construcción de la masculinidad en tres hombres saltillenses a partir de relatos de vida, pues para Bertaux (1999), los relatos de vida cumplen tres funciones dentro del análisis social y a su vez, ayudan a cumplir con el objetivo establecido en este trabajo de investigación: en primer lugar, se plantea que los relatos de vida realizan una función exploratoria, logrando iniciar al investigador en un campo específico. En segundo lugar, los relatos, son utilizados para el sostén de una teoría frente a la vida real y, por último, utilizan la síntesis como un recurso para la recolección y asociación de los datos obtenidos en los relatos con el trabajo teórico.

Para lograr objetivo se tomaron como objetivos específicos:

1. Identificar las prácticas sociales que influyen en la construcción de la masculinidad en las narrativas sobre el cuerpo y la indumentaria de tres hombres saltillenses.

2. Identificar las pautas culturales que influyen en la construcción de la masculinidad en las narrativas sobre el cuerpo y la indumentaria de tres hombres saltillenses. 
3. Analizar cómo influyen las prácticas sociales y pautas culturales de la masculinidad en las narrativas sobre el cuerpo y la indumentaria de tres hombres saltillenses.

\section{Marco teórico}

\subsection{Construyendo el género en sus prácticas sociales y pautas culturales}

Establecer la idea de observar el género como resultado de una construcción social, presupuso entretejer una relación que permitiera identificar el proceso por el cual el género es construido en la cotidianidad. Por ello, este trabajo se apoyó a nivel conceptual de las reflexiones hechas por Berger y Luckmann (2003) en su obra teórica de La Construcción Social de la Realidad y de algunas de las posturas de la teoría de género hechas por Lamas (2015), Rubin (1986) y Scott (1986). Este entretejido teórico permitió situar el género como producto de una invención social que se produce y reproduce, por medio de las interacciones que hacen los sujetos sociales de manera subjetiva y colectiva en la vida cotidiana a manera de conocimiento. Dicho conocimiento se muestra objetivado por medio del lenguaje y la visualidad, pues estos sistemas de comunicación permiten significar y tipificar la relación sistema sexo-género. Ello a partir de las ideas establecidas en un determinado espacio histórico social sobre el cómo debe de comportarse una persona, cómo debe de relacionarse, lo que el mundo espera de ella y cómo debe visualizarse ante los demás a partir de su género.

Estos cúmulos de conocimiento construyen, dan significado y vuelven interpretable, la subjetividad de cada individuo a partir de sus prácticas sociales (Castro et al., 1996) y las pautas culturales de Geertz (1975) o Guerrero (2002), adscritas por el orden social de género. En este sentido, se argumenta que la forma en la que el sujeto construye su subjetividad determina si representa un sujeto acorde a su realidad o a una otredad dentro del sentido común de la vida cotidiana.

\subsection{La construcción social de la masculinidad y sus narrativas del cuerpo y la indumentaria}

Entender que la masculinidad no es atribuida de forma natural por aspectos biológicos, sino construida socialmente durante la interacción de los individuos en una cultura específica, da pie a reflexionar que esta construcción no sucede de manera armónica. Lo anterior, al funcionar como un filtro de lo que debe y se espera sea un hombre (Lamas, 2015), sirve como un sistema regulador que controla y cohesiona los cuerpos y sus prácticas. En este tenor, se considera que la constitución de la masculinidad en la subjetividad de cada persona significa una batalla frente a un orden social, al representar fronteras simbólicas que son impuestas y muchas veces adoptadas y reproducidas por el hombre en su cotidianidad por medio de sus prácticas (Connel, 1995).

Estos procesos de construcción subjetiva se ven regulados por un formato normativo de género a través del cual las sociedades condicionan cómo debe ser un varón para ser considerado tal, a partir de dos creencias: la prescriptiva como aquello que debe hacerse para ser un hombre, y la proscriptiva, como lo que no puede hacerse para ser hombre (Bonino, 2001). Estas divisiones categóricas comúnmente se ven reguladas por la masculinidad hegemónica que provoca que valores como la autosuficiencia, rechazo de emociones, fuerza física, superioridad hacia lo que no es masculino y la heterosexualidad, se conviertan en ideales intrasubjetivos que todos los varones deben alcanzar (Bonino, 2001). Dichas creencias son producidas y reproducidas de manera colectiva, siendo la homosociabilidad entre los propios hombres (Kimmel, 1994) el medio por el cual se transmiten los "significados masculinos" que deben ser reproducidos en la subjetividad de cada individuo como orden social (Berger y Luckmann, 2003).

Sin embargo, para Connel (1995) esta serie de pautas y valores adscritos a la masculinidad no se encuentra estructurado de la misma manera, pues al ser una construcción social, la representación ideal del hombre cambia según el modelo predominante que se genera en las relaciones de género de un contexto específico. Así pues, se provoca que el formato normativo atribuido a la masculinidad no sólo cree prácticas y relaciones asociadas al valor hegemónico, sino muchas otras variaciones como la masculinidad subordinada, la masculinidad relacionada a la complicidad y la masculinidad marginal; este último modelo, para el autor, es construido a partir de la interrelación entre el género $\mathrm{y}$ diversos atributos que forman parte de la subjetividad como la clase, la edad, la raza y la experiencia de vida de cada individuo. Haciendo así que surjan múltiples relaciones 
amplias y complejas sobre el significado de ser, comportarse y vivir como un hombre.

Al tomar una postura en la que se creé existen múltiples modelos de masculinidad que son construidos socialmente en la subjetividad de cada individuo y que son justamente estos aspectos subjetivos del hombre, como el cuerpo y la vestimenta, los que lo posicionan como un agente normal o una otredad, se decidió basar la investigación en las narrativas del cuerpo y la indumentaria de las masculinidades marginales de las que habla Connel (1995). Este modelo refleja el dinamismo y la plasticidad del género como producto de una construcción social a partir de las distintas formas en la que los hombres construyen y viven su masculinidad, al mismo tiempo que representan, desde su subjetividad, las múltiples realidades que conforman los distintos hilos con los que se crea el tejido social de género.

En este marco, Zambrini (2010) expone que asumir los modos de vestir desde una dimensión discursiva, supone entender la importancia del leguaje como generador de significados en donde se construyen y se hacen objetivos los valores culturales y normativos de género. Zambrini (2013) nombra a estos discursos como "narrativas sobre las indumentarias y las corporalidades", en dónde el cuerpo vestido es capaz de narrar la forma en la que se construyen los géneros social y culturalmente en un determinado espacio social e histórico. Esta propuesta fue retomada durante la investigación cómo narrativas del cuerpo y la indumentaria y fue asociada con la subjetividad masculina, al considerar que, además de narrar estos procesos de construcción masculina en un lugar específico, también relatan la manera en la que los hombres construyen su masculinidad desde sus subjetividades, la forma en la que perciben, reproducen o contradicen el performance de género de la masculinidad (Butler, 1990), además de narrar datos significativos que van conformando la experiencia de los hombres en la vida cotidiana a través de la indumentaria, los movimientos, los gestos, los adornos y las marcas corporales.

\section{Propuesta metodológica}

Teniendo en cuenta lo anterior, se consideró una propuesta metodológica de enfoque cualitativo que permitiera responder a la pregunta y objetivos planteados en esta investigación.
Desde el punto de vista metodológico, el trabajo se adscribe al enfoque biográfico (Aceves, 1998), esto desde una perspectiva en la que no se trate el relato sólo como palabra, sino también desde la visualidad (Banks, 2008), dando la posibilidad de observar los múltiples discursos que se encuentran presentes en estos procesos de construcción a partir de los relatos de vida (Bertaux, 1989) y la fotobiografía (Cronin, 1998) como las técnicas utilizadas en esta investigación.

En cuanto la muestra con la que se decidió trabajar, el interés principal se centró en estudiar la subjetividad de hombres de la ciudad de Saltillo, Coahuila, en México, que correspondieran a lo que Connel (1995) considera como el hombre marginal dentro del orden social de la masculinidad. Este término también es abordado por Plummer (1986) quien propone la selección de sujetos cuya subjetividad se encuentre construida a partir de aspectos sociales y culturales distintos a los de la norma. Se decidió trabajar con tres hombres, pues se cree que al estudiar las subjetividades que representan lo marginal no es necesario contar con una muestra basada en una representatividad estadística, sino una muestra a conveniencia que a partir de lo que exponen Mallimaci y Giménez (2006), sea elegida por medio de criterios teóricos y conceptuales que resulten relevantes para responder a la pregunta y objetivos de investigación.

Por esta razón, se realizó una búsqueda que permitiera localizar a tres hombres cuyos cuerpos, la forma en la que los adornan y visten, correspondieran a la otredad que caracteriza al hombre marginal frente a las prácticas entendidas como naturales, e ideales de un hombre (Connel, 1995), en este caso, de la ciudad de Saltillo. A partir de este punto de referencia, de una distribución de rangos etarios dentro de la muestra que permitiera observar los distintos significados de ser hombre, que se construyen a lo largo de la experiencia (Mallimaci y Giménez 2006), y de la técnica de bola de nieve, se pudo localizar a: Don Armando, un maestro de la sastrería de 81 años de edad que se caracteriza por ser uno de los últimos sastres que se mantienen activos en la ciudad de Saltillo y que, al vestir de casimir, destaca frente a la forma en la que comúnmente se viste en Saltillo. Según él, la industrialización de la ciudad y las modas que se siguen hoy en día, han provocado que se pierda el arte y el gusto del buen vestir. El segundo es Patricio, estudiante de derecho de 22 años que trabaja como mesero en un popular restaurante de la ciudad 
para pagar sus estudios y su tratamiento de cambio de sexo. Él es un hombre transgénero que utiliza los movimientos de su cuerpo y su vestimenta para demostrar que es un hombre. La tercera persona es Vampi, un padre de familia de 29 años quien es conocido en la ciudad como el vampiro de Saltillo por aspectos particulares de su cuerpo e indumentaria que forman parte de un estilo de vida acorde al vampirismo.

A partir de los objetivos y la metodología planteada en este proyecto, y de las recomendaciones hechas por Mallimaci y Giménez (2006) sobre la estructura del eje temático y la guía del relato, se decidió usar dos técnicas de investigación que permitieran documentar la información durante el trabajo de campo: la entrevista semiestructurada y la observación participante. Estas técnicas de recolección ayudaron a realizar un acercamiento a la vida cotidiana de los informantes, a sus rutinas, a las narraciones sobre sus experiencias de vida y a su forma de llevar y vestir el cuerpo, con la finalidad de construir los relatos que dieran cuenta del proceso de construcción bajo el cual significan la masculinidad con relación a las narrativas del cuerpo y la indumentaria.

En cuanto el periodo de tiempo estipulado para realizar el trabajo de campo y el número de visitas planeadas para los encuentros con los informantes, se consideró un total de 15 sesiones; cinco por cada informante con una duración de 60 minutos aproximadamente, todas ellas distribuidas periódicamente cada tercera semana. Ello con la intención de cubrir cuatro sesiones por mes en un periodo de tres meses, correspondiente a mayo-agosto de 2017.

Una vez documentada la información recuperada durante el trabajo de campo, se propuso analizar dicha información a partir de tres modelos de análisis ayudaron a responder a los objetivos y la pregunta planteada en esta investigación. En este tenor, el primer sistema de análisis fue diseñado a partir de la realización y el uso de un diario de campo (Valverde, 1996) como modelo de recolección y análisis desde una actitud reconstituyente Demazière y Dubar (citado en Pretto, 2011), en donde se facilitaron las transcripciones en extenso de las sesiones de charla, además de presentar un reporte acerca de lo acontecido en cada uno de los encuentros, y aunado a una tabla de información con anotaciones hechas durante las sesiones. Para la realización del segundo sistema de análisis se apoyó de la propuesta de Demazière y Dubar (citado en Pretto, 2011) sobre la actitud ilustrativa. En este segundo sistema se seleccionaron y clasificaron fragmentos de las entrevistas en tablas de información que permitieran dar cuenta de las prácticas sociales y las pautas culturales que se identificaron en los relatos, así como los temas en los que se adscriben estas prácticas y pautas.

Finalmente, se apoyó de la propuesta hecha por Bertaux (citado en Pretto, 2011) sobre el análisis comprensivo para la realización del tercer modelo de análisis, en donde se dedicó a contestar la pregunta de investigación planteada en este proyecto, a partir de la construcción de tres relatos interpretativos, mismos que ayudaran a comprender cómo construyen estos tres hombres su masculinidad, subjetivamente, con relación a las narrativas del cuerpo y la indumentaria. La escritura de estos relatos fue estructurada bajo un orden cronológico, con la intención de observar de manera ordenada de qué manera sucedió este proceso de construcción que se pudo identificar y analizar a lo largo del relato de nuestros informantes.

A partir de algunos aspectos que fueron identificados en el relato de los informantes, en donde el contexto social se presentó como un factor determinante dentro del proceso de construcción de su masculinidad, y de las reflexiones hechas por Berger y Luckmann (2003), Geertz (1973), Zambrini (2013), Butler (1990) y Connel (1995), acerca del papel que cumple el espacio histórico social en la conformación de la vida cotidiana y el género, se consideró necesario realizar una caracterización de Saltillo. Lo anterior, con el fin de poder observar de qué manera se presenta socialmente el lugar en el que los informantes articulan su cotidianidad. Esta caracterización se realizó a partir de libros históricos, textos científicos y documentos como fotografías y ediciones de periódicos. Así se pudo deducir que el trabajo industrial, la presencia de la religión católica y la construcción de marcados roles de género significan tres factores importantes que forman parte de la vida cotidiana de la Saltillo, mismas que se encuentran presentes en el relato de Don Armando, Patricio y Vampi.

Con la intención de observar los discursos visuales (Villalplana, 2015) presentes en las narrativas del cuerpo y la indumentaria de los informantes, se realizó una sesión fotográfica con cada uno de ellos con el fin de capturar, a través de la imagen, los discursos que construyen cotidianamente por medio de su cuerpo vestido en un entorno visual. Estas series 
de imágenes fueron trabajadas desde la actitud restituyente de Demazière y Dubar (citado en Pretto, 2011) con la intención de que sus relatos hablados, y las narrativas del cuerpo y la indumentaria que se presentan por medio de la visualidad, se complementaran y brindaran información en conjunto sobre los procesos subjetivos de llevar y vestir el cuerpo en la cotidianidad.

\section{Resultados}

A partir del análisis hecho con la información obtenida durante el trabajo de campo, y de la construcción de tres relatos interpretativos, uno por cada informante, fue posible realizar una serie de interpretaciones acerca del proceso por el cual los colaboradores del proyecto han construido y construyen su masculinidad desde su subjetividad. Asimismo se pudo interpretar el papel que toman sus narrativas del cuerpo y la indumentaria en el proceso. Esta serie de interpretaciones se desarrollaron de manera independiente por cada informante, pues al suponer una primera exploración al proceso subjetivo de hacerse hombre, se consideró necesario dar valor a aquellas experiencias que han significado en la vida de Don Armando, Patricio y Vampi de forma separada.

A continuación, se presentan algunas de las reflexiones finales realizadas en este proyecto de investigación sobre el proceso de construcción de la masculinidad y el papel que toma la subjetividad en él. Del mismo modo, se exponen algunas de las complicaciones metodológicas que se presentaron durante el trabajo de campo y su respectiva interpretación.

Durante los encuentros realizados con el señor Armando, el tema principal en el que se desenvolvió cada charla estuvo relacionado con su experiencia dentro de la sastrería. A la par de estas anécdotas, Don Armando repetía constantemente su posicionamiento acerca de la importancia que toma el buen vestir en su trabajo y en otras profesiones, además de demostrar su desagrado con la forma en la que las personas visten hoy en día, en específico, en el uso cotidiano de la mezclilla.

A partir de esto se pudo comprender, o al menos se interpretó de esta manera, que cada una de las experiencias que compartió el informante estaban relacionadas con el oficio de la sastrería pues este supone un cúmulo de conocimiento
(Berger y Luckmann, 2003) a partir del cual Don Armando ha constituido su realidad. Se considera que esto sucede por el papel que ha tomado la sastrería en su vida, ya que este oficio ha estado presente a lo largo de su aquí y ahora, formando parte importante dentro del proceso de construcción de su masculinidad:

[...] yo no tuve infancia, no me dejaron. No tuve niñez, me la cortaron bien. Pues de primero no me gustaba esta madre. Le decía a mi madre: -no, a mí no me gusta esto-, -¿por qué?-, -pues no me gusta-. Pero no aquí está, pues se acostumbraba a lo que mi padre le daba y pues ahí te quedas. Duré muchos años de pantalonero, muchos años. Siempre pensando: al rato me salgo, al rato me voy, lo voy a dejar al otro día, pero no. Luego ya me fui para México, vino mi cuñado y nos fuimos para allá. Pero antes de casarme, vine a ver a mi madre y me dice mi cuñado el que me enseñó -Necesitas aprender a hacer sacos porque no vas a poder mantener a la mujer, de pantalonero no-. (Don Armando, Comunicación personal, 5 de junio 2017)

En el caso de Patricio, fue posible observar un factor que se considera parte importante en el proceso de construcción de la masculinidad que hace desde su subjetividad, pues muchas de las experiencias que narró sobre su vida cotidiana fueron relacionadas por él mismo con algunos conceptos de la teoría de género y la masculinidad. Se considera que el conocimiento que posee sobre estas temáticas conforma parte de su vida cotidiana al suponer, en su caso, un cúmulo de conocimiento ligado con su condición como hombre transgénero y que vuelve objetiva su masculinidad (Berger y Luckmann, 2003).

Esto propició que cada uno de los relatos se mantuviera centrado en su proceso de construcción, aceptación y transformación como hombre transgénero, en el cual fue posible destacar tres momentos clave en donde el cuerpo y la vestimenta ocuparon un papel significativo: su infancia, en donde se concibió a él mismo como un niño; su adolescencia, en donde se identificó como una mujer lesbiana y, por último, en su aceptación y estilo de vida como hombre transgénero.

[...] cuando creces y ya tienes la oportunidad de decidir y tienes la visión de decir esta es la ropa 
que yo quiero usar y verte y verte en el espejo y verte como hombre, realmente como lo que eres, te hace sentir seguro, te da paz, te sientes conforme con lo que ves, que aunque no es como quisieras, pero te ves y hasta dices -este soy yo[...] el poderme vestir de esa manera y que pase desapercibido y que la gente te trate como él, porque vas vestido, vas realmente proyectando lo que eres y anteriormente no era así, anteriormente era usar ropa de niña y siempre con la cabeza agachada [...] para mí el vestirme de niña era estar amarrado, odiaba los vestidos rotundamente los odio. (Patricio, Comunicación personal, 1 de septiembre 2017)

En cuanto Vampi, el tema principal presente en las sesiones de charla fue su vida cotidiana como vampiro, estilo de vida que práctica desde su juventud. En estas charlas se pudo apreciar que las distintas experiencias que ha afrontado como hombre han estado acompañadas junto al proceso en el que se ha constituido como un vampiro, en donde las narrativas del cuerpo y la indumentaria toman un rol significativo, al ser las distintas modificaciones que ha hecho a su cuerpo y su vestimenta los atributos que significan estas experiencias. De esta forma, el colaborador construyó su relato a partir de los distintos momentos en los que se ha significado como vampiro y hombre a la vez, proceso en el que su familia, sus amistades, la música y su desinterés por adscribirse a lo normal forman parte importante:

[...]a mi punto de vista es que un vampiro viene de mucho más atrás y sí tiene mucha vida, tiene que estar acoplándose con toda la gente, con todos los géneros, tiene uno que aprender, no nada más se tiene que quedar en el rock o en lo suyo, no nada más se tiene que quedar en el vampirismo y ya [...] Mi hermano siempre fue de que -Vete más atrás y vete más atrás. ¿Qué es lo que te gusta? - No pues el vampirismo-Vete más atrás, vete más atrás, ya cuando estés ahí no de que el vampiro es, es esto y lo otro, de que tiene vida eterna, metete, porque vas cambiando de año, en un año cambia esto, en un año cambia otra cosa, en un año cambian géneros, las personas, la vestimenta, pues tienes que acoplarte a ellos. Un vampiro se supone que tiene que estar mezclado entre la gente, para que no te vea mezclado, o sea, que no te descubran que eres el vampiro que llevas por dentro, al contrario que te vean como una persona normal. (Vampi, Comunicación personal, 04 de noviembre de 2017)

Para Berger y Luckmann (2003) la identidad es un elemento clave de la realidad subjetiva que se construye de forma dialéctica con los procesos sociales que se encuentran determinados por una estructura social, siendo, en otras palabras, construida por medio de la conciencia individual y la estructura social; el individuo y la sociedad; el cuerpo y la identidad social. Tal perspectiva constituye los resultados presentados en esta aproximación a la subjetividad masculina, en donde justamente, son estos dos términos, subjetividad y masculinidad, los hilos que entretejen los relatos de Don Armando, Patricio y Vampi; sus experiencias, sus emociones, parte de su identidad y con ello, sus narrativas del cuerpo y la indumentaria.

A partir de lo anterior, se cree que este tejido no es sólo una aproximación a la construcción de la masculinidad, sino también el proceso por el que se constituye la experiencia de cada sujeto desde su subjetividad. Es en estas trayectorias de la cotidianidad en donde el género se presenta objetivo a partir de una serie de simbolizaciones que se muestran visibles en el gesto, el movimiento corporal, el vestido y el significado del cuerpo. Esto se pudo observar en cada una de las experiencias compartidas por los informantes y que están relacionadas con sus narrativas sobre el cuerpo y la indumentaria. En ellas, sus cuerpos vestidos significaron cada una de las experiencias que afrontaron en su vida cotidiana al percibirse como hombres. En este sentido, se puede destacar la dialéctica bajo la cual se construyen estas narrativas en donde el cuerpo es moldeado en la experiencia de cada sujeto, de sus emociones y de su identidad, con el fin de significarse, identificarse y diferenciarse de los demás a través de su movimiento, su adorno y su vestido.

Es necesario abordar en esta última parte del documento la situación que estuvo presente en las sesiones realizadas con Don Armando y Vampi, en relación a la incomodidad 
que mostraron cuando se abordó el tema sobre el significado que atribuyen a su cuerpo. Se cree que la negativa ante estos temas forma parte del desapego que tiene el hombre de hablar sobre su cuerpo con otros hombres al mostrarse como temas de índole privado, pues, al no formar parte de los temas cotidianos que se tratan en lo público, estas charlas son relacionadas con la feminidad. Por otra parte, es posible relacionar esta situación con otro factor que se presentó durante el trabajo de campo, en específico, durante la realización del proyecto fotográfico, en el cual se percibió a Don Armando y a Patricio nerviosos ante el hecho de posar para la sesión fotográfica. Al igual que la negativa que mostraron Vampi y Don Armando con el abordaje de temas como la masculinidad o el cuerpo. Es posible asumir que tal situación también forma parte del contexto específico en el que se desarrolló este proyecto, en donde la homosociabilidad entre hombres se limita sólo a observar y modelar los cuerpos para tipificarse e identificarse entre sí como hombres.

Se considera que el trabajo de tesis realizado supone una primera aproximación a las subjetividades en el que se pudo constatar el papel que toma el género, así como en el espacio histórico-social, el cuerpo, la indumentaria y la visualidad en la nunca acabada construcción de las subjetividades que se producen, se mantienen y se modifican conforme se significa y se mueve el cuerpo en la cotidianidad.

Así, resulta necesario nombrar estas reflexiones como in-conclusiones; resulta complicado concluir algo que apenas inicia, al considerar que este ejercicio de observación e interpretación, realizado para responder a la pregunta planteada en el proyecto de tesis, abre a más preguntas y posibilidades para abordar las narrativas del cuerpo la indumentaria, la subjetividad, la visualidad y el género en futuras investigaciones, pues la masculinidad, al ser moldeable, puede serlo todo menos un proceso estático y acabado.

\section{Referencias}

Aceves, J. (1995). La historia oral y de vida: del recurso técnico a la experiencia del investigador. En M. Calderon (Ed.), Técnicas de investigación en sociedad, cultura y comunicación (pp.207-276). Naucalpan de Juárez, México: Pearson.
Banks, M. (2010) Los datos visuales en Investigación Cualitativa. Madrid, España: Ediciones Morata.

Berger, L y Luckmann, T (2003)La construcción social de la realidad. Recuperado de https://zoonpolitikonmx.files.w ordpress.com/2014/09/la-construccic3b3n-social-de-la -realidad-berger-luckmann.pdf

Bertaux, D. (1989). Los relatos de vida en el análisis social. Historia y Fuente oral, 1, 136-148. Recuperado de https: //www.jstor.org/stable/27753230

Bertaux, D. (1999). El enfoque biográfico: su validez metodológica, sus potencialidades. Proposiciones, 29 , 1-23. Recuperado de www.sitiosur.cl/publicaciones/Re vista_Proposiciones/PR-0029-3258.pdf

Bonino, L. (2001) La masculinidad tradicional, obstáculo a la educación en igualdad. En Galicia, X (Presidencia) La masculinidad tradicional, obstáculo a la educación en igualdad. Conferencia llevada a cabo en Congreso Nacional de Educación en Igualdad, Santiago de Compostela, España.

Butler, J. (1990). Actos performativos y constitución del género: un ensayo sobre fenomenología y teoría feminista. Desde la Teoría 1, 296-314. Recuperado de http://www.debatefeminista.cieg.unam.mx/wpcontent /uploads/2016/03/articulos/018_14.pdf

Castro, P., Chapman, R., Gilli, S., Lull, V., Mico, R., Rihuete, C., Rish, R. y Sanahuja, E. (1996). Teoría de las prácticas sociales. Compliutum Extra, 6(2) 35-48. Recuperado de http://asome.uab.cat/Teoria\%20de\%201as\%20practica s\%20sociales2.pdf

Connel, R. (1995). La organización social de la masculinidad. En Olavarria, J. (Ed). Masculinidad/es: poder, crisis (pp. 31-48). Ciudad de México, México: Ediciones de las Mujeres. Recuperado de http://higualitaris.grunyi.net/ wordpress/wp-content/uploads/organizacion-social-m asculinidad-connell.pdf

Cronin, O. (1998). Psichology and photographic theory. En Prosser, J. (Ed.). Image-based research: a sourcebook for quealitative researches (pp. 120-159). Londres, Inglaterra: Faimer Press.

Demazière, D. y C. Dubar. 1997. Analyser les entretiens biographiques. Paris, Editions Nathan.

Geertz, C. (1973). La interpretación de las culturas. Recuperado de: https://antroporecursos.files.wordp ress.com/2009/03/geertz-c-1973-la-interpretacion-delas-culturas.pdf

Giménez, G. (2012). Comunicación, cultura e identidad. Reflexiones epistemológicas. Cultura $y$ Representaciones Sociales, 6(11), 109-132. Recuperado de http://www.scielo.org.mx/pdf/crs/v6n11/v6n11a5.pdf Guerrero, P. (2002). La cultura. Estrategias conceptuales para entender la identidad, la diversidad, la alteridad y la diferencia. Recuperado de http://digitalrepository.unm. edu/abya_yala/10/ 
Kimmel, M. (1994). Masculinity as Homophobia. Recuperado de http://www.suarakita.org/wp-content/uploads/2016/ 02/masculinity.pdf

Lamas, M. (marzo de 2015). Repensando la masculinidad. En A. Güezmes (Presidencia), Conversatorio dentro del mes de la igualdad de género. Conferencia llevada a cabo en el Conversatorio dentro del mes de la igualdad de género. Ciudad de México, México.

Mallimaci, G. y Giménez, V. (2006). Historia de vida y métodos biográficos. En Vasilachis, I. (Ed). Estrategias de investigación cualitativa (pp. 175-209). Barcelona, España: Gedisa.

Plummer, K. 1986. Documents of Life. An Introduction to the Problems and Literature of a Humanistic Method. Londres: Allen \& Unwin.

Pretto, A. (2011). Analizar las historias de vida: reflexiones metodológicas y epistemológicas. Tabula Rosa, 15, 171-194.

Rubin, G. (1986). El tráfico de mujeres: Notas sobre la "economía política" del sexo. Nueva Antropológica, 8(30), 95-145. Recuperado de www.caladona.org/g rups/uploads/2007/05/El\%20trafico\%20de\%20mujeres 2.pdf

Scott, J. (1986). El género: una categoría útil para el análisis histórico. En Lamas, M. (Ed.). El género: la construcción cultural de la diferencia sexual (pp. 265-302). Ciudad de México, México: PUEG.

Valverde, A. (2009) El diario de Campo. Binass 11, 308-319. Recuperado de http://www.binasss.sa.cr/revistas/ts/v18n 391993/art1.pdf

Villaplana, V. (septiembre de 2015). Metodologías visuales para la investigación en las ciencias sociales. En Escala, L. (Presidencia) Seminario Metodologías visuales para la investigación en ciencias sociales. Seminario llevado a cabo en: Colegio de la Frontera Norte. Baja California, México.

Zambrini, L. (2010). Modos de vestir e identidades de género: reflexiones sobre las marcas culturales en el cuerpo. Nomadias, (11). doi:10.5354/0719-0905.2010.15158

Zambrini, L. (2013). El género como metáfora: narrativas sobre travestis en prensa digital argentina (2004-2009). Sociedad y Economía, 24, 143-58. Recuperado de revist aingenieria.univalle.edu.co/index.php/sociedad_y_eco nomia/article/.../6126/ 